\title{
Spatial Pattern Coding of Sensory Information by Climbing Fiber-Evoked Calcium Signals in Networks of Neighboring Cerebellar Purkinje Cells
}

\author{
Simon R. Schultz, ${ }^{1,2,3}$ Kazuo Kitamura, ${ }^{2}$ Arthur Post-Uiterweer, ${ }^{1}$ Julija Krupic, ${ }^{1}$ and Michael Häusser ${ }^{2}$ \\ ${ }^{1}$ Department of Bioengineering, Imperial College London, South Kensington, London SW7 2AZ, United Kingdom, ${ }^{2}$ Wolfson Institute for Biomedical \\ Research and Department of Neuroscience, Physiology and Pharmacology, University College London, London WC1E 6BT, United Kingdom, and ${ }^{3}$ Gatsby \\ Computational Neuroscience Unit, University College London, London WC1N 3AR, United Kingdom
}

\begin{abstract}
Climbing fiber input produces complex spike synchrony across populations of cerebellar Purkinje cells oriented in the parasagittal axis. Elucidating the fine spatial structure of this synchrony is crucial for understanding its role in the encoding and processing of sensory information within the olivocerebellar cortical circuit. We investigated these issues using in vivo multineuron two-photon calcium imaging in combination with information theoretic analysis. Spontaneous dendritic calcium transients linked to climbing fiber input were observed in multiple neighboring Purkinje cells. Spontaneous synchrony of calcium transients between individual Purkinje cells falls off over $\sim 200 \mu \mathrm{m}$ mediolaterally, consistent with the presence of cerebellar microzones organized by climbing fiber input. Synchrony was increased after administration of harmaline, consistent with an olivary origin. Periodic sensory stimulation also resulted in a transient increase of synchrony after stimulus onset. To examine how synchrony affects the neural population code provided by the spatial pattern of complex spikes, we analyzed its information content. We found that spatial patterns of calcium events from small ensembles of cells provided substantially more stimulus information ( $59 \%$ more for seven-cell ensembles) than available by counting events across the pool without taking into account spatial origin. Information theoretic analysis indicated that, rather than contributing significantly to sensory coding via stimulus dependence, correlational effects on sensory coding are dominated by redundancy attributable to the prevalent spontaneous synchrony. The olivocerebellar circuit thus uses a labeled line code to report sensory signals, leaving open a role for synchrony in flexible selection of signals for output to deep cerebellar nuclei.
\end{abstract}

\section{Introduction}

Multiple-electrode recording studies have shown that Purkinje cell spontaneous complex spiking (CS) activity is synchronized in narrow bands along the rostrocaudal axis of the cerebellum on a millisecond timescale (Bell and Kawasaki, 1972; Sasaki et al., 1989). The functional significance of this synchrony is not clear, although an increase in synchrony during skilled movement (Welsh et al., 1995) and after electrical stimulation of motor cortex (Schwarz and Welsh, 2001) suggests a role in coordinating motor behavior. To understand the functional role of CS synchrony for sensorimotor behavior, however, we must address the following key issues: first, the fine-scale spatiotemporal structure

\footnotetext{
Received 0ct. 9, 2008; revised May 7, 2009; accepted May 16, 2009.

S.R.S. was supported by Engineering and Physical Sciences Research Council Grants GR/S85467 and EP/ E002331/1 and by the Royal Society and the Gatsby Charitable Foundation. K.K. was supported by the Japan Society for the Promotion of Science. M.H. was supported by the Wellcome Trust and the Gatsby Charitable Foundation. We thank Benjamin Judkewitz, Séverine Mahon, Arnd Roth, and Phil Bream for useful discussions and David Schoppik, Mickey London, Alexandre Mathy, and Christian Wilms for their comments on this manuscript.

Correspondence should be addressed to either of the following: Simon R. Schultz, Department of Bioengineering, Imperial College London, South Kensington, London SW7 2AZ, UK, E-mail: s.schultz@imperial.ac.uk; or Michael Häusser, Wolfson Institute for Biomedical Research, University College London, London WC1E 6BT, UK, E-mail: m.hausser@ucl.ac.uk.

K. Kitamura's present address: Department of Neurophysiology, Graduate School of Medicine, University of Tokyo, 7-3-1, Hongo, Bunkyo-ku, Tokyo 113-0033, Japan.

D0I:10.1523/JNEUROSCI.4919-08.2009

Copyright $\odot 2009$ Society for Neuroscience $\quad$ 0270-6474/09/298005-11\$15.00/0
}

of synchrony; second, how sensory stimulation shapes synchronous ensembles; and third, how it affects sensory coding.

Multiple-electrode arrays typically have an intersite spacing of several hundred micrometers, and, thus, whereas CS correlation and its relationship to the anatomical zonal structure of the cerebellum is understood on a broad spatial scale (Sugihara et al., 2007), the fine-scale spatial correlation structure on the level of neighboring neurons is unknown. To examine our first question, we thus ask how synchrony falls off with mediolateral distance between cells.

To understand the role of the cerebellum in sensorimotor integration, we must know whether and how CS synchrony depends on sensory stimulation. A motor role is suggested by a study showing absence during passive sensory responses but presence during a tongue-extension movement (Welsh, 2002). Others have emphasized that climbing fibers are activated synchronously in response to unexpected sensory consequences of motor actions (Lou and Bloedel, 1992). In anesthetized rat Crus IIa and rabbit vestibulocerebellum, sensory stimulation has been found to modulate CS synchrony (Llinás and Sasaki, 1989; Wylie et al., 1995). These results leave open how sensory stimulation spatiotemporally shapes the prevalent spontaneous synchrony.

The third crucial question concerns how sensory signals are combined across neurons. Is the spatial pattern of activity important, or just the number of events? If the identity of the neuron 
firing a spike "tags" the spike with some informational value, we refer to this as a "labeled line" code. In contrast, in a "pooled" code, spikes have the same meaning regardless of their cellular origin and spatial information is neglected. In a labeled line code, correlations might further lead to coding by combinatorial patterns across cells. To test which of these three population coding strategies is used, we quantify the information present in both pooled spike count and spike pattern codes, and we ask how much of this information is attributable to spatial correlations.

We have addressed these questions using two-photon imaging of calcium signals in the dendrites of multiple Purkinje cells bulkloaded with AM ester calcium dye (Stosiek et al., 2003). By calibration with simultaneous electrophysiology, we show that this technique allows us to examine the spatiotemporal dynamics of the CS sensory population code. We find that calcium signals in nearby Purkinje cells are synchronized well above chance levels, with synchrony falling off over several hundred micrometers mediolaterally. We also find that somatosensory stimulation increases synchrony during a brief period (up to several hundred milliseconds) after stimulus onset. Finally, we examine the fidelity of the CS population code for the timing of stimulus onset, finding that the spatial pattern of CS activity carries considerably more information than is available by counting spikes.

\section{Materials and Methods}

The care and experimental manipulation of animals was performed in accordance with the regulations of the United Kingdom Home Office.

Surgery. Sprague Dawley rats (postnatal days 18-29) were anesthetized with urethane $(1.2 \mathrm{~g} / \mathrm{kg})$ or with a ketamine $(50 \mathrm{mg} / \mathrm{kg})$-xylazine $(5$ $\mathrm{mg} / \mathrm{kg}$ ) mixture. The level of anesthesia was routinely monitored by observing whisker movements and/or monitoring the hindlimb legwithdrawal reflex and supplementary doses of anesthetic administered if required. A craniotomy was made to expose folium Crus IIa, the dura was carefully removed, and a stainless steel frame was glued to the skull with dental cement. The craniotomy was filled with $1.5-2 \%$ agarose in Ringer's solution, and a coverslip was clamped above the agarose to suppress brain movement, leaving an opening for microelectrode access (Svoboda et al., 1999). Body temperature was maintained at $37-38^{\circ} \mathrm{C}$ using a heating blanket.

In vivo multiphoton imaging. Bulk loading of AM ester calcium indicator dye was performed using a protocol similar to those described previously (Stosiek et al., 2003; Sullivan et al., 2005; Gao et al., 2006). Briefly, for each experiment, 1 aliquot $(50 \mu \mathrm{g})$ of Oregon Green BAPTA-1 (OGB-1) AM calcium indicator dye (Invitrogen) was dissolved in DMSO containing 20\% w/v Pluronic F-127 (Invitrogen) and diluted 3:100 in a buffer solution containing $150 \mathrm{~mm} \mathrm{NaCl}, 2.5 \mathrm{~mm} \mathrm{KCl}$, and 10 mM HEPES, $\mathrm{pH} 7.4$, to give a final dye concentration of $300 \mu \mathrm{M}$ OGB-1 AM. Alexa 594 was also added to the solution to aid visualization of dye ejection (final concentration, $10 \mu \mathrm{M}$ ). A micropipette was filled with this solution and inserted into the cerebellar molecular layer (depth typically $100-150 \mu \mathrm{m}$ below pia). Dye was ejected by applying pressure at $40 \mathrm{kPa}$ for $\sim 1$ min using a Picospritzer (General Valve) or by repeated brief puffs with a $5 \mathrm{ml}$ syringe.

Imaging was performed $>30 \mathrm{~min}$ after dye ejection, using a twophoton laser scanning microscope (Prairie Technologies). Excitation was provided by a pulsed titanium/sapphire laser system operating at $810 \mathrm{~nm}$ (Mai Tai; SpectraPhysics) with $<100$ fs pulse width and $80 \mathrm{MHz}$ repetition rate, focused using a $40 \times, 0.8$ numerical aperture objective lens (Olympus). Images were acquired using ScanImage (Pologruto et al., 2003) for MATLAB (MathWorks). For every region imaged, a highresolution reference image was first acquired $(512 \times 512$, average of five frames), followed by a spontaneous activity run (typically $256 \times 64$ or $256 \times 32$ resolution, $1000-2000$ frames, $128 \mathrm{~s}$ of data) and, if appropriate, a series of one to five sensory-evoked runs (typically 500-1000 frames, $64 \mathrm{~s} \mathrm{each).} \mathrm{Raster} \mathrm{lines} \mathrm{making} \mathrm{up} \mathrm{each} \mathrm{frame} \mathrm{were} \mathrm{of} 2$ or $2.3 \mathrm{~ms}$ duration, resulting in frame rates of $7-16 \mathrm{~Hz}$. In some experiments, harmaline was administered by intraperitoneal injection $(10 \mathrm{mg} / \mathrm{kg})$ as described by Lamarre et al. (1971). Imaging resumed 7-10 min after harmaline injection.

Electrophysiology. Targeted extracellular recordings were made from imaged neurons using patch pipettes $(\sim 4 \mathrm{M} \Omega)$ filled with artificial CSF and containing Alexa 594. The pipette was navigated until the tip was adjacent to a Purkinje cell soma or dendrite and complex spikes could be detected with high signal-to-noise ratio (SNR). Electrophysiological and imaging data were then simultaneously acquired from the same Purkinje cell; identification of regions containing calcium signals corresponding to electrophysiological complex spike activity was performed offline.

Sensory stimulation. Brief air-puff stimuli (50 psi, $50 \mathrm{~ms}$ ) were applied to the perioral surface or ipsilateral whiskers via a glass tube mounted on a micromanipulator, timed by a Picospritzer. Sensory-evoked trials consisted of a nonstimulated period of $12-15 \mathrm{~s}$, followed by a periodic train of $20-32$ puffs separated by $1.28-3.68 \mathrm{~s}$.

Data analysis. Regions of interest (ROIs) corresponding to individual Purkinje cells were identified offline via three methods described in the supplemental data (available at www.jneurosci.org as supplemental material): human operator-driven identification based on both a time-series movie and high-resolution reference image, pixel cross-correlation maps, and spatial independent component analysis (Reidl et al., 2007). All three methods resulted in pixel clusters lining up in a narrow rostrocaudal plane, with qualitative agreement in area definition. Fluorescence time series corresponding to the average intensity of pixels within an ROI were extracted. Cross-covariance and joint poststimulus time histogram (JPSTH) analyses were performed directly on the fluorescence timeseries traces. Calcium transient event trains were obtained from timeseries traces by applying a custom-written template-matching algorithm, the performance of which was verified by simultaneous electrophysiology (supplemental Fig. S3, available at www.jneurosci.org as supplemental material).

Information theory. Information calculations were performed using a binary code produced by the template-matching procedure, in which a bin took the value of 1 if it corresponded to the beginning of a template match and 0 otherwise. Responses, $r$, were formed via either of two methods. For spike pattern codes, the response $\mathbf{r}=\left\{r_{i}\right\}$ was the vector of single cell responses $r_{i}$, where $i$ is the cell index; for spike count codes,

$$
r=\sum_{i} r_{i}
$$

is in this special case a scalar quantity. The spike pattern response code thus had a cardinality of $2^{C}$, where $C$ is the number of Purkinje cells, and the spike count code had a cardinality $Q+1$, where $Q$ is the maximum count of 1 values observed from individual ROIs on any trial. One key difference between these codes is that, for spike pattern codes, the identity (spatial location) of the cell firing a spike matters, whereas for spike counts, only the pooled number of events across the population can carry information. The conditional response distribution matrices $P(\mathbf{r} \mid s)$ were formed, where $s$ indicates the time bin relative to stimulus onset from which the responses were drawn. In a reduced version of the calculation to compute signal detection information, only two such response time bins were used to form the conditional response matrix: one before stimulus onset and one at the peak response time. Results were similar using both approaches, and, for the sake of generality, the former has been presented in this study. The mutual information for both spike pattern and spike count codes was obtained by computing the difference between a total entropy $H(R)$ and a noise entropy $H(R \mid S)$ :

$$
I(R ; S)=H(R)-H(R \mid S)
$$

where

$$
H(R)=-\sum_{\mathbf{r}} P(\mathbf{r}) \log _{2} P(\mathbf{r})
$$

with $H(R \mid S)$ similar but for the conditional distribution $P(\mathbf{r} \mid s)$ and averaged over stimuli $s$.

To analyze the contributions of different aspects of the statistical struc- 
ture of neural population activity to the information, we broke the total pattern information down into a number of information components that reflect different contributions to neural coding: $I=I_{\text {ind }}+I_{\text {cor,ind }}+$ $I_{\text {cor,dep }}$. We calculated the information that would be conveyed by a fictional ensemble of neurons with identical firing rate profiles but zero "noise correlation" (correlation across neurons of the response variability from trial to trial with the same stimulus, as is induced by synchrony). This can be calculated by shuffling the stimulus-conditional response trials separately for each neuron. However, calculating this information quantity amounts to replacing $P(\mathbf{r} \mid s)$ with

$$
P_{\text {ind }}(\mathbf{r} \mid s)=\prod_{i} P\left(r_{i} \mid s\right)
$$

(Schneidman et al., 2003; Pola et al., 2003; Montemurro et al., 2007), and direct implementation of the latter expression is more efficient in sampling terms. Comparing the value $I_{\text {ind }}$ obtained by inserting this distribution into the mutual information equation with the total information allows us to measure the overall effect of correlations (which can be positive or negative) on the amount of information conveyed. The component of the information attributable to the effect of correlations can be calculated as $I_{\text {cor }}=I-I_{\text {ind }}$.

There are, however, a number of ways in which noise correlation can affect information transmission. For instance, stimulus modulation of synchrony might be used explicitly as an information channel. The contribution of this mechanism to information coding can be measured as

$$
I_{\text {cor,dep }}=I-\sum_{i} \sum_{r_{i}}\left\langle P\left(r_{i} \mid s\right) \log _{2} P\left(r_{i} \mid s\right)\right\rangle_{s}+\sum_{\mathbf{r}} P(\mathbf{r}) \log _{2} P_{\text {ind }}(\mathbf{r}),
$$

where

$$
P_{\text {ind }}(\mathbf{r})=\sum_{s} P(s) P_{\text {ind }}(\mathbf{r} \mid s)
$$

This quantity is identical to the $\Delta I$ measure proposed for the information loss to a decoder in neglecting correlations (Nirenberg et al., 2001). The average level of correlation can, however, also affect fidelity of information transmission, either improving or decreasing it (Panzeri et al., 1999); this can easily be measured as $I_{\text {cor,ind }}=I_{\text {cor }}-I_{\text {cor,dep. }}$. We thus break the total pattern information into a number of information components that reflect different contributions to neural coding: $I=I_{\text {ind }}+I_{\text {cor,ind }}+$ $I_{\text {cor,dep. }}$.

Another comparison we can make is to compare the total pattern information with that obtained by linear summation of the information from each cell separately,

$$
I_{\operatorname{lin}}=\sum_{i} I\left(R_{i} ; S\right)
$$

This comparison examines not just the effect of noise correlation but also of similarity of tuning (signal correlation); in fact, $I_{\text {ind }}=I_{\text {lin }}+I_{\text {sig-sim }}$, where $I_{\text {sig-sim }}$ is a negative correction to $I_{\text {ind }}$ attributable to the similarity of signals conveyed. If the pattern information is greater than the sum of the single-cell information, the coding is synergistic; if less, the information carried by each cell is on average redundant. We normalize this to calculate a redundancy fraction (Gawne et al., 1996):

$$
\text { Fractional redundancy }=\frac{I_{\mathrm{lin}}-I}{I} .
$$

Entropies were estimated using the Nemenman-Shafee-Bialek algorithm (Nemenman et al., 2004). Our information-theoretic analysis procedures have been described in more detail previously (Montani et al., 2007). One hundred to 200 trials per stimulus were typically available for entropy estimation. Supplemental Figure S5 (available at www.jneurosci.org as supplemental material) demonstrates that this provides adequate sampling for both spike count and pattern codes with the dimensionalities examined. For the results shown in Figure 5B, animals under ketamine/xylazine and urethane anesthesia were combined, because the mean information values were similar in both cases. The mutual information contained by responses about the time of their occurrence relative to stimulus onset was computed. The robustness of our information estimates with respect to finite sampling bias are shown in supplemental Figure S5 (available at www.jneurosci.org as supplemental material).

Results are given as mean \pm SEM unless otherwise indicated.

\section{Results}

Purkinje cells in cerebellar folium Crus II were loaded with OGB-1 AM and imaged using two-photon microscopy (Fig. 1 and supplemental Movie 1, available at www.jneurosci.org as supplemental material) (Stosiek et al., 2003; Sullivan et al., 2005). Spontaneous dendritic calcium transients were observed at a rate of $\sim 1 \mathrm{~Hz}$. To identify the origin of the dendritic signals, we made simultaneous targeted extracellular recordings from the respective Purkinje cells using a patch pipette containing Alexa 594 (Fig. 1 and supplemental Movie 2, available at www.jneurosci.org as supplemental material). ROIs corresponding to individual Purkinje cells were determined using time-series fluorescence movies (supplemental Movie 3, available at www.jneurosci.org as supplemental material) (see Materials and Methods). As shown in Figure $1 C$, each complex spike observed on the electrophysiological trace corresponds to an event in the selected ROI. When a pair of CS occurred in rapid succession, a single large dendritic calcium transient was observed, indicating that CS rate observed using our approach $(0.58 \pm 0.01$ and $1.02 \pm 0.03 \mathrm{~Hz}$ using ketamine/xylazine or urethane anesthesia, respectively) represents a slight underestimate, which nevertheless is consistent with previous results using two-photon imaging (Sullivan et al., 2005), as well as conventional electrophysiological approaches (Ito, 1984). The observed CS rates are also similar to those observed in the awake rat (Lang et al., 1999). We conclude that the overwhelming majority of the detected spontaneous dendritic calcium transients under our conditions reflect spontaneous climbing fiber input.

We next examined the degree of spontaneous CS synchrony across nearby Purkinje cells up to $200 \mu \mathrm{m}$ apart. Figure 2, $A$ and $B$, shows an experiment in which spontaneous activity was imaged in dendrites from 15 different Purkinje cells. The fluorescence time series for the Purkinje cell dendritic regions were obtained (Fig. 2C), and cross-covariance between these time series were calculated to quantify synchronicity of spontaneous CS between each Purkinje cell pair (Fig. 2D). The dendrites of neighboring Purkinje cells show partial synchrony (mean crosscovariance, $0.32 \pm 0.01$ for 569 pairs located up to $25 \mu \mathrm{m}$ apart mediolaterally), decreasing with mediolateral separation (Fig. $2 E)$. Cross-covariance declined over several hundred micrometers (Fig. $2 \mathrm{~F}$ ) (decay constant, $225 \mu \mathrm{m}$; 95\% confidence interval, 189-261 $\mu \mathrm{m}$; pooled results from 2235 pairs of Purkinje cells under ketamine/xylazine and urethane anesthesia). If the synchrony observed is the result of gap junctional coupling between inferior olive cells, it should increase after administration of harmaline, which enhances subthreshold oscillations and network synchrony in the olive (Llinás and Yarom, 1986). Indeed, harmaline significantly increased CS synchrony (Fig. $2 G)(70 \pm 0.2 \%$; $n=164$ pairs from 5 experiments), consistent with previous results using electrophysiological techniques (Llinás and Sasaki, 1989; Sasaki et al., 1989; Lou and Bloedel, 1992; Welsh et al., 1995) with lower spatial resolution.

We triggered sensory input using brief perioral air-puff stimuli (Brown and Bower, 2002; Chadderton et al., 2004). When sensory-evoked calcium signals were detected (Fig. $3 A$ ), neighboring cells showed varying sensory response reliability: some responded to nearly every stimulus (e.g., the seventh cell in Fig. 
A

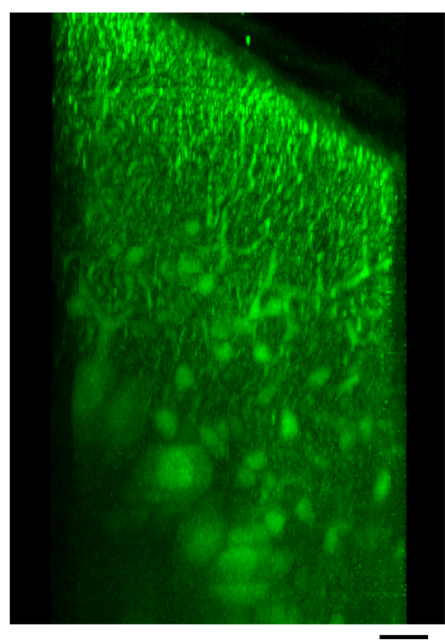

B

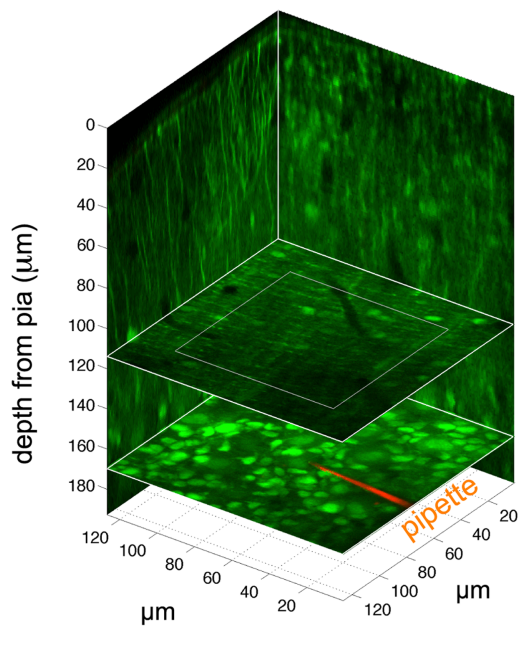

C

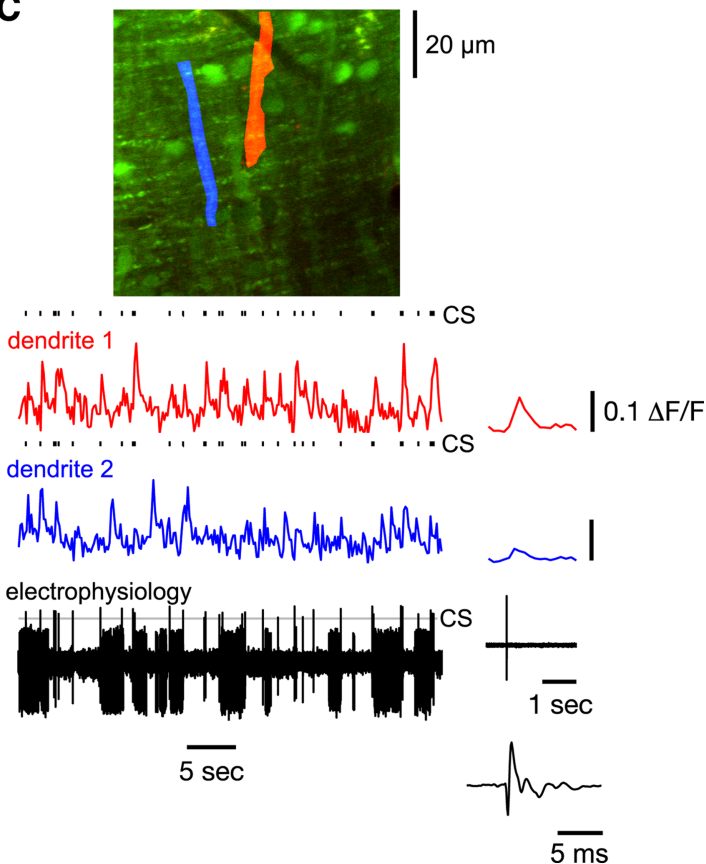

Figure 1. Two-photon imaging of climbing fiber-evoked calcium signals in Purkinje cell dendrites. $A$, Side projection of a three-dimensional image stack ( $251 \mu \mathrm{m}$ total depth) showing in vivo staining of cerebellar Purkinje cell dendrites by pressure ejection of the dye 0GB-1 AM. B, Three-dimensional slice reconstruction of an experiment in which two-photon targeted extracellular recording was performed in the Purkinje cell layer, followed by calcium imaging in the molecular layer above. The green channel shows OGB-1 labeling, and the red shows the pipette containing Alexa 594. For clarity, the bottom slice has been replaced by a maximum-intensity projection through the surrounding $20 \mu \mathrm{m}$ of tissue. C, Calcium imaging of the region indicated by the inner gray square in $\boldsymbol{B}$. Of the two regions of interest defined here, one (red) corresponds to the recorded cell. The electrophysiological trace at the bottom contains both simple spikes and climbing fiber-evoked CS, the latter defined by crossing the threshold (gray line) and marked above the calcium traces by tick marks (repeated identically above both traces for convenience). In the red trace, there is a one-to-one relationship between calcium transients and the recorded CS; the same is not true for the second region (blue trace). This results in a strong $C S$-triggered fluorescence waveform for the red region (at right), with the blue region showing a smaller CS-triggered average attributable to the presence of regional CS synchrony. Bottom right, Expanded timescale showing the average CS waveform observed extracellularly.

$3 A$ ), whereas others (with equally large calcium transients) were more sporadic (first and fourth cells). Sensory stimulation at similar $(0.3-0.8 \mathrm{~Hz})$ rates to spontaneous CS rate did not significantly increase the time-averaged rate of calcium transient events across cells (Kolmogorov-Smirnov test; $n=113 ; p=0.17$ ), as shown in Figure $3 C$. This held whether comparing template-match event rates or the underlying rate of fluorescence fluctuations (supplemental Fig. S3, available at www.jneurosci.org as supplemental material). Given the presence of clear sensory-locked responses visible in single cells, this suggests that the sensory stimulus results in entrainment of CS activity (Marshall and Lang, 2004) rather than the production of additional complex spikes.

CS trains can be described as a highly irregular renewal process under spontaneous conditions (Shin et al., 2007). How is this affected by sensory stimulation? We computed the coefficient of variation statistic $\left(\mathrm{CV}_{2}\right)$ interspike interval (ISI), equal to the SD divided by the mean of the distribution of successive ISIs, which measures the local irregularity of interspike intervals (Holt et al., 1996). We found that interevent intervals were more regular under stimulated than spontaneous conditions (Fig. 3D) (twosample Kolmogorov-Smirnov test, $p=7 \times 10^{-8}$ ). Although this might be expected as a natural consequence of regular stimulation, it does suggest that more reliable CS temporal coding may be possible than is suggested by stochastic descriptions based on spontaneous activity.

As a result of sensory entrainment, a high degree of synchrony would be expected simply because of each neuron in a pair tending to respond to the stimulus at a similar time; this is what we observed (Fig. $3 E$ ). Correcting for this effect by subtracting a shift predictor, the time-averaged cross-correlogram was observed to be not significantly different under sensory-driven compared with spontaneous conditions (two-sample Kolmogorov-Smirnov test, $p>0.2 ; n=70$ pairs with SNR $>0.4$; urethane anesthesia). However, this leaves open the possibility that synchrony is temporally modulated, which could mean that spatial patterns occurring at particular times relative to stimulus onset might convey information.

To examine the effect of sensory stimulation on CS synchrony in more detail, we computed the normalized JPSTH (nJPSTH) (Fig. 4) (Aertsen et al., 1989). Approximately half of the responding pairs of cells were found to have greater sensory-modulated synchrony than expected by chance ( 21 of 43 pairs with geometric mean SNR exceeding 0.2; Student's $t$ test, $p<0.05$ ). The $t$ test used to assess this is a conservative statistical measure. The example shown in Figure $4 A$ is typical, showing a brief period of strong synchrony shortly after stimulus delivery. The average nJPSTH for pairs of cells with geometric mean of the SNRs of each cell exceeding 0.2 is shown in Figure $4 B$. Although these pairs do not all show the same time dependence of either synchrony or firing rate, common features are apparent across the dataset, such as modulation along the JPSTH diagonal after stimulus onset, and less joint events than expected (from the PSTH marginals) at longer asynchronies $(>0.4 \mathrm{~s})$. Pairs containing at least one member unresponsive to the stimulus instead show an nJPSTH reflecting "spontaneous synchrony": an unmodulated diagonal band in the JPSTH, with no suppression on the flanks of the crosscorrelogram (Fig. $4 C$ shows the average for pairs with geometric mean SNR <0.05). These effects are unlikely to be accounted for 
A

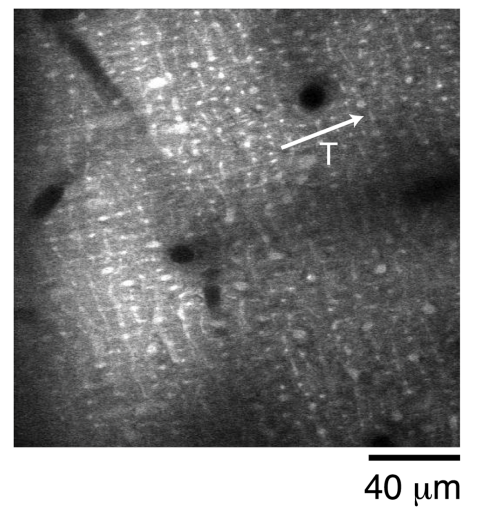

B

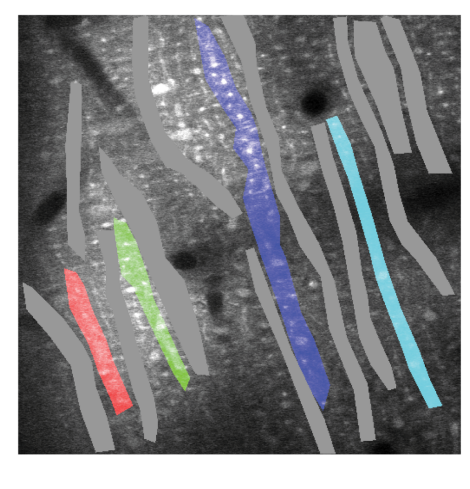

C
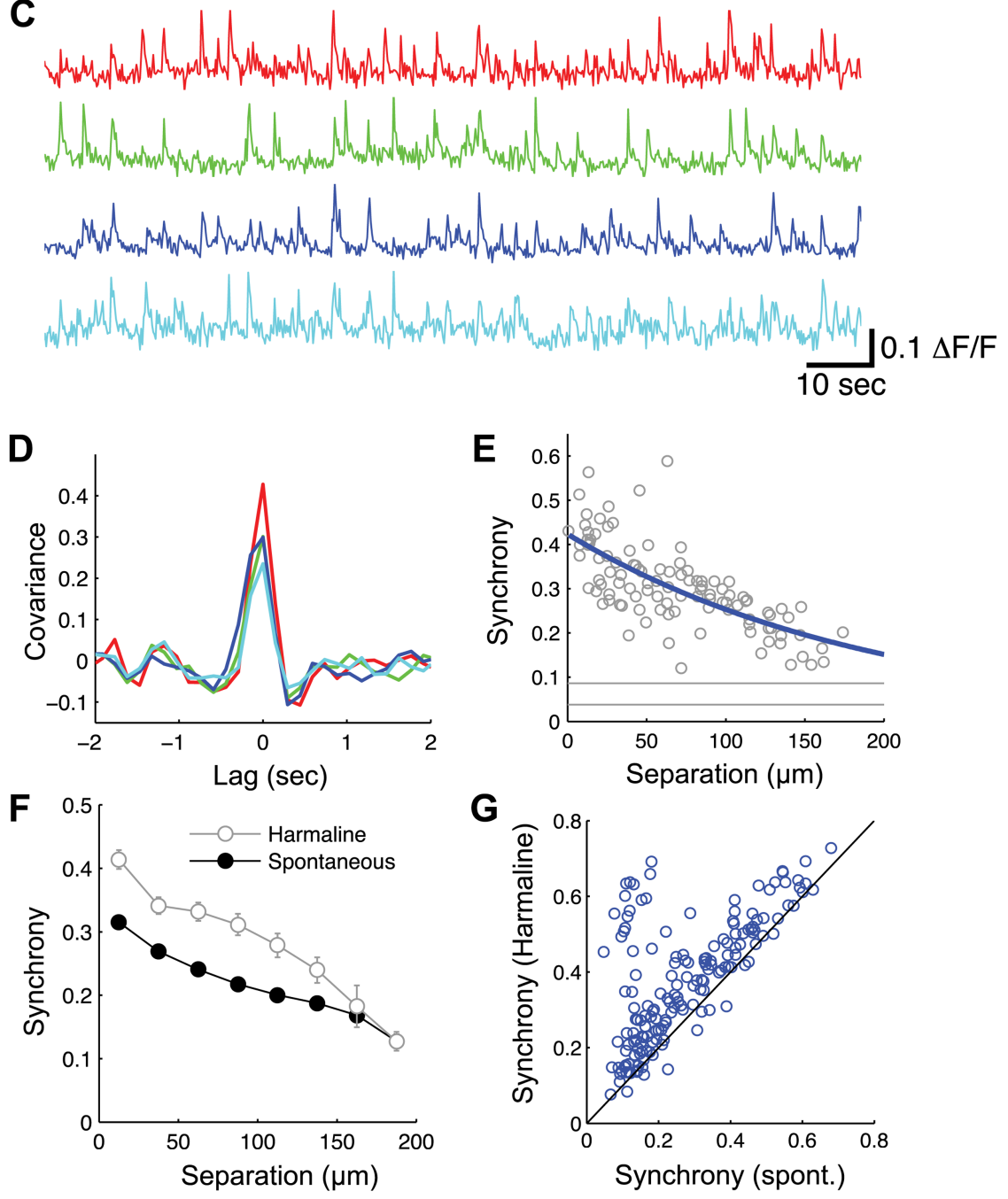

G

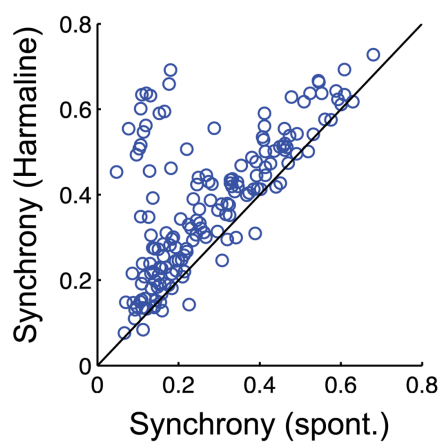

Figure 2. Synchrony in dendritic calcium signals falls off over $\sim 200 \mu \mathrm{m}$ mediolaterally. $A$, A plane in the molecular layer of Crus lla (depth of $\sim 50 \mu \mathrm{m}$ ). The transverse axis is marked by the arrow labeled T. $\boldsymbol{B}$, ROls were defined using the procedures described in the supplemental data (available at www.jneurosci.org as supplemental material); this required the use of time-series movies in addition to the reference images shown. $C$, Time series for four Purkinje cell dendritic regions (colors correspond to $B$ ) show decreasing likelihood of sharing fluctuations with transverse separation. $\boldsymbol{D}$, Cross-covariance of the four traces in $C$ with the trace for the leftmost ROl indicates that zero-lag covariance (synchrony) is reduced with mediolateral distance between cells. $\boldsymbol{E}$, Synchrony for all pairs in $\boldsymbol{B}$ plotted against separation between dendrites. Gray lines indicate $90 \%$ confidence intervals for the cross-covariance expected by chance, established by generating surrogate time-series data with shuffled event times (see supplemental data, available at www.jneurosci.org as supplemental material). Solid curve shows a single-exponential fit (spatial decay constant, $195 \mu \mathrm{m}$ ). $\boldsymbol{F}$, Population average transverse falloff of synchrony for 2235 pairs from 27 animals. Error bars shown indicate SEM and, when not visible, are obscured by symbols. Filled symbols, Spontaneous synchrony; open symbols, after administration of harmaline. $\mathbf{G}$, Data from experiments in which the same region was imaged before and after harmaline injection ( $n=7$ from 5 animals). by residual sensory modulation attributable to inadequate correction for PSTH effects, because this would be reflected in a correlation between the nJPSTH magnitude and the amplitude of stimulusinduced responses, and no such correlation was evident (supplemental Fig. S4, available at www.jneurosci.org as supplemental material). The sensory effect on synchrony, as measured by the peak height of the correlation poststimulus histogram (CPSTH), was dependent on mediolateral distance (correlation coefficient, -0.37 ; $n=123$ pairs; significant at $p<0.001$ ) (Fig. 4D), as found for spontaneous synchrony. The space constant of decay was $186 \mu \mathrm{m} \quad(95 \%$ confidence interval, 102-270 $\mu \mathrm{m})$, comparable with that observed for spontaneous synchrony.

The modest stimulus dependence of CS synchrony we observed raises the question of whether modulation of synchrony (or of CS patterns of which the synchrony may be a second-order signature) may convey significant sensory information beyond that available to a downstream deep cerebellar nucleus (DCN) neurons simply by counting the spikes fired by the input neurons in a short time window. Addressing this question requires knowledge of, first, whether patterns of CS in a local area matter or just their total number (i.e., making the distinction between pattern and pooled codes), and, second, whether the important aspects of those patterns relate to synchrony or to cell identity (in the latter case, we have a very particular case of the pattern code, the labeled line code, in which downstream neurons can make use of the identity of the neuron firing each spike but in which a combinatorial code across neurons is not used). There are thus three population coding approaches that might be used: pooled coding, labeled line coding, and more general pattern coding. Explicit sensory modulation of synchrony might a priori be expected to lead to the latter. We therefore examined the information contained in calcium signals from local populations of neurons about when responses occurred relative to stimulus onset, i.e., information about stimulus timing. The higher this quantity, the more accurately the local population represents the time of stimulus occurrence. This mutual information quantity is bounded by the stimulus entropy, here determined by the temporal precision with which responses are binned, for example, with a frame duration of $64 \mathrm{~ms}$ (typically used in our study), the stimulus entropy including time bins from 0.0 to $1.0 \mathrm{~s}$ after stimulus onset is 4 bits. 

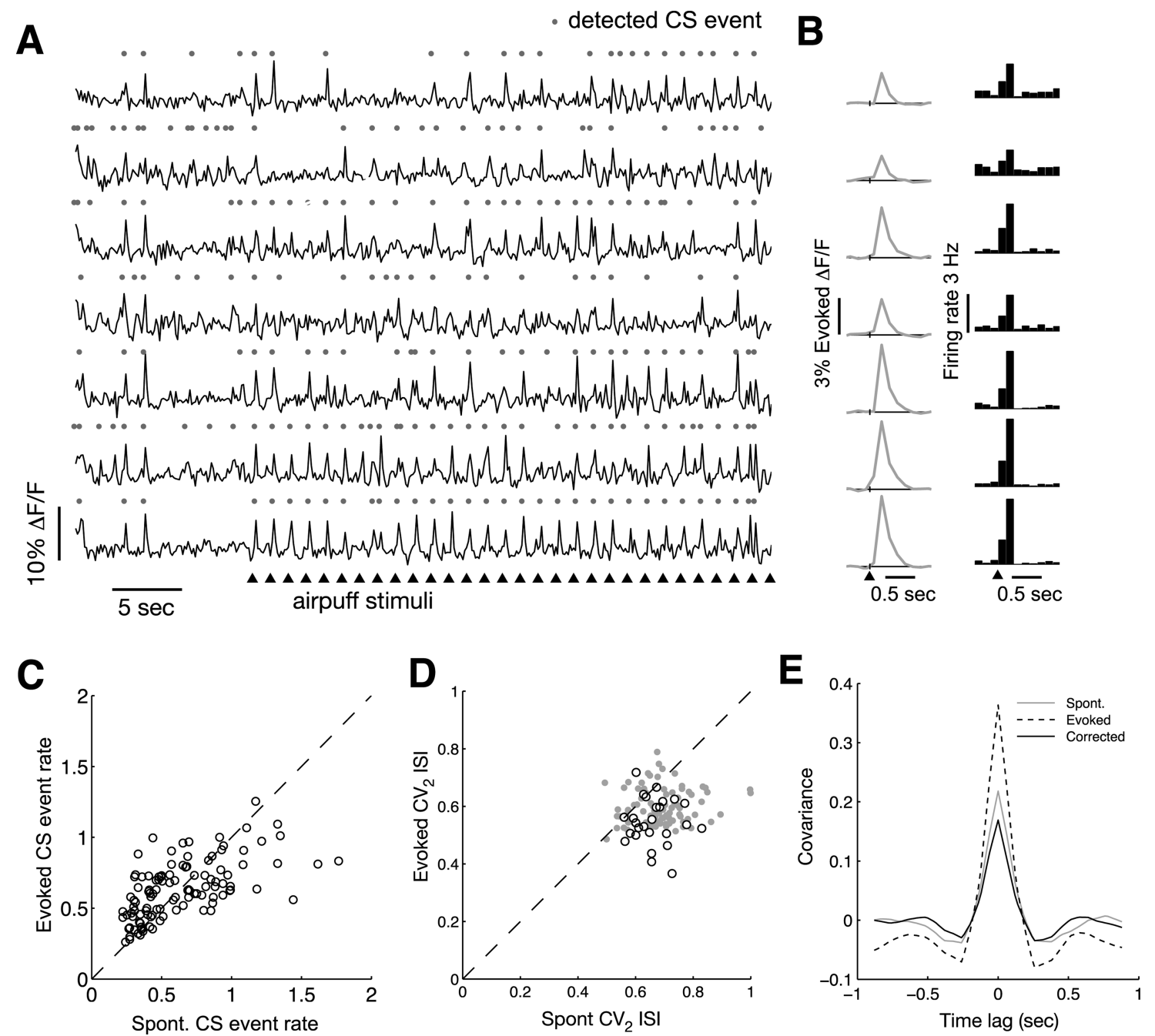

Figure 3. Sensory stimulation entrains climbing fiber-evoked activity. $\boldsymbol{A}$, Fluorescence time-series traces for a region in which ROIs corresponding to seven Purkinje cells have been identified. Triangles indicate air-puff stimuli applied to the lower lip every $1.28 \mathrm{~s}$. Imaging frame rate is 8 frames $/ \mathrm{s}$ for this example, and the traces have been high-pass filtered (first-order Bessel filter, cutoff of $0.4 \mathrm{~Hz}$ ). Calcium transient events detected using a template-matching algorithm have been indicated above the time series by gray dots. $\boldsymbol{B}$, Average fluorescence increase evoked by air-puff stimulation for each of the regions indicated in $\boldsymbol{A}$ (at left) and PSTH for calcium transient events (at right). $\boldsymbol{C}$, Calcium transients evoked by climbing fiber activity occur at the same rate under sensory-induced and spontaneous activity conditions. Cells shown were selected according to the criterion that the sensory signal-to-noise ratio exceeds 0.1 . D, Sensory stimulation increases the reliability of interspike intervals, leading to a reduction in $\mathrm{CV}_{2} \mid \mathrm{SI}$. Gray dots indicate cells with $\mathrm{SNR}>0.1$, and open circles indicate cells with $\mathrm{SNR}>0.4$ (strongly responding). $\boldsymbol{E}$, Under sensory-driven conditions, the time-averaged level of synchrony increases, but this can be accounted for by stimulus locking.

We computed the information carried by events detected using the template-matching procedure from ensembles of single Purkinje cell ROIs as described in the supplemental data (available at www.jneurosci.org as supplemental material). Figure $5 \mathrm{~A}$ shows the information carried by ensembles of up to seven of the dendrites shown in Figure 3, for both spike pattern and spike count codes. In this population of neurons, a more reliable estimate of stimulus timing is available to a receiver (e.g., DCN neurons) capable of distinguishing between different spatial patterns of its inputs than to one restricted to counting the number of active inputs without taking into account their origin (pooling). This result cannot be explained by finite sampling bias differentially affecting these two population codes, because the 200 trials per stimulus available were more than adequate to sample both codes with the entropy estimation approach used (supplemental Fig. S5, available at www.jneurosci.org as supplemental material). The result held across the dataset ( $n=8$ animals), with on average $59 \%$ additional information available from patterns in the activity of seven-cell ensembles $(n=9)$ (Fig. $5 B, C)$.
What is the origin of this spatial pattern information? A clue is provided by comparing the pattern information with that provided by responses that have been shuffled to destroy any stimulus-conditional (noise) correlations but preserve firing rate profiles (we refer to this information value as $I_{\text {ind }}$; see Materials and Methods). We found that the information conveyed is higher when correlations are destroyed than when they are present (Fig. $5 A, C$, green lines; $D)$. This suggests that, despite the presence of a modest amount of stimulus dependence of the correlations (Fig. 4), the overall effect of the prominent CS synchrony is a reduction in the total information transmitted. This is surprising because synchrony is an obvious candidate code for reading out the time of occurrence of the sensory stimulus (but see Discussion). This result, together with that of the previous paragraph, would appear to fit with a labeled line picture of cerebellar CS population coding.

$I_{\text {ind }}$ captures the component of the total information that is preserved if noise correlations are neglected. However, some forms of correlation can still affect $I_{\text {ind }}-$ signal correlations, which 
A Correlation coefficient \begin{tabular}{ccccc}
-0.2 & 0 & 0.2 & 0.4 & 0.6 \\
\hline & & & &
\end{tabular} $\sec$

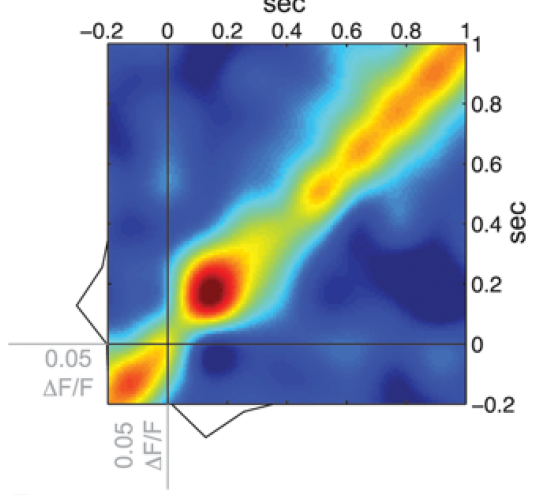

B

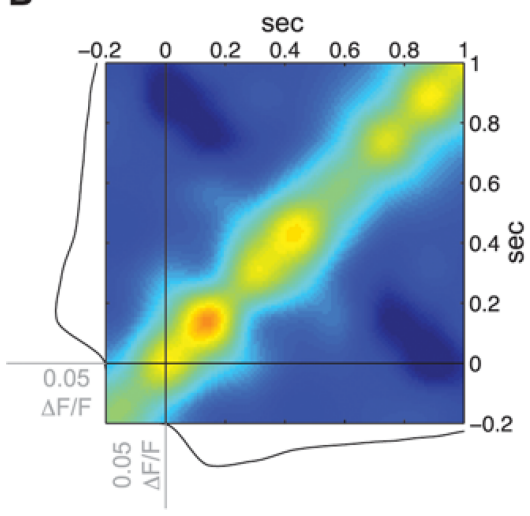

C
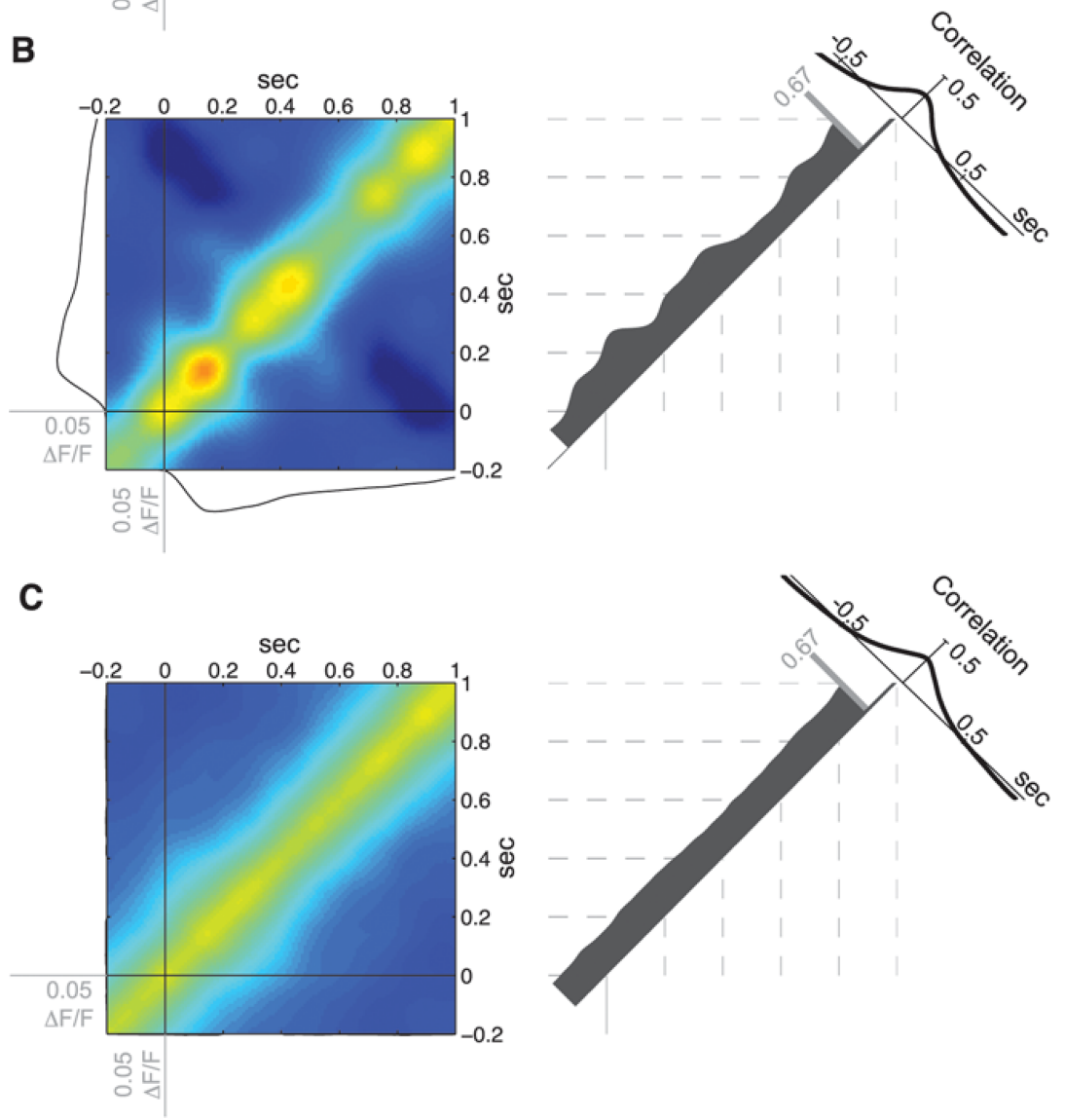

D

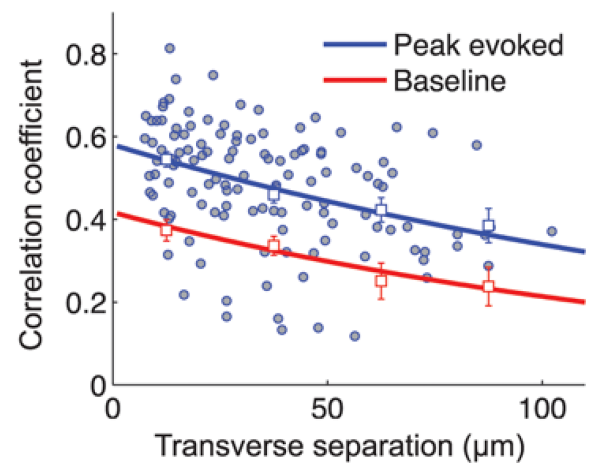

Figure 4. Sensory stimulation temporally modulates synchrony of calcium signals in nearby Purkinje cells. A, nJPSTH for a typical pair of cells (third and fifth examples from Fig. $3 A$ ), showing synchronous activity immediately after stimulus onset, beyond that expected attributable to individual responses. The stimulus-triggered average fluorescence response of each dendrite is shown at bottom and left; at right is shown the CPSTH from the diagonal of the nJPSTH. Averaging instead across time yields the may be thought of as correlations between the tuning profiles of the cells. If there is neither signal correlation nor noise correlation, then we have "informational independence," in which the information available from each cell can simply be added linearly to obtain the total ensemble information. This distinction between conditional response independence (as captured by the quantity $I_{\text {ind }}$ ) and informational independence has been made previously (Schneidman et al., 2003). Our results show that the pattern information is both lower than what would be expected from response independence (Fig. 5D) and lower than what would be expected from informational independence (Fig. $5 E)$; both noise and signal correlations have the effect of reducing the information that can be transmitted.

We can analyze the contributions of different aspects of pattern correlations to neural coding in more detail using information component analysis (Panzeri et al., 1999; Panzeri and Schultz, 2001; Pola et al., 2003; Montani et al., 2007), which involves breaking up the total pattern information into contributions that do and do not depend on noise correlations: $I=I_{\text {ind }}+I_{\text {cor }}$. For a labeled line code, $I_{\text {ind }}$ might be expected to dominate, but nevertheless if the lines were correlated, there would be an unavoidable effect on the information, which would be captured by $I_{\text {cor. }}$ Examining the distributions of these quantities computed for pairs of neurons (Fig. 6A), we observe that, whereas $I_{\text {ind }}$ contributes the most to the total information $(0.129 \pm$ 0.008 bits; $n=77$ pairs for which geometric mean SNR >0.2), the correlational contribution $I_{\text {cor }}$ is almost always negative and distributed around small negative values $(-0.018 \pm 0.002$ bits $)$. To examine exactly how this correlational contribution arises from the interplay between the average level of correlation [which can either

expectation-corrected cross-correlogram. Scaling is such that all correlation quantities can be interpreted as Pearson's coefficients. Gray bar on CPSTH indicates height of peak in this plot. $\boldsymbol{B}$, The average nJPSTH for 103 pairs of cells with SNR $>0.2$. CPSTH and cross-correlogram on same scale as $\boldsymbol{A}$. $\boldsymbol{C}$, The average JPSTH for 430 pairs of cells, of which neither responded strongly to the sensory stimulus (SNR $<0.05$ ), shows only spontaneous synchrony. D, Peak CPSTH values reflecting stimulus-evoked correlation for each pair of cells fall off with transverse separation between the cells. Filled circles, Peak $n J P S T H$ for all pairs with geometric mean SNR $>0.2$. Blue open squares, Mean in $25-\mu \mathrm{m}$-wide bands. Solid blue line, Single-exponential fit. Red symbols and line show a similar result for the baseline period $0.4-0.2 \mathrm{~s}$ before stimulus onset. Error bars indicate SEM. 
increase or reduce the information (Panzeri et al., 1999)] and stimulus dependence of the correlation (which may either increase the information or have no effect), $I_{\text {cor }}$ can be broken down further, into terms $I_{\text {cor,ind }}$ and $I_{\text {cor,dep }}$ respectively. $I_{\text {cor,ind }}$ captures the effect of the average level of correlation on information transmission (which is unavoidable in the sense that it affects a downstream receiver regardless of whether it is explicitly optimized for detecting correlation or not). In contrast, $I_{\text {cor,dep }}$ captures the contribution to the information from explicit sensory modulation of the correlation, such as might occur as a result of sensorymodulated synchrony or to use of the structure of a combinatorial spatial pattern code to convey information. Figure $6 \mathrm{~A}$ shows that, in this case, the contribution of correlations to the information is almost entirely dominated by the average level of correlation $\left(I_{\text {cor,ind }}=-0.028 \pm\right.$ 0.003 bits and $I_{\text {cor,dep }}=0.010 \pm 0.001$ bits, respectively).

An important question that arises is whether the role of interactions between Purkinje cells (such as synchrony) in sensory coding differs according to whether the cells are nearby or farther apart. Figure $6 B$ shows a breakdown of the results described above according to mediolateral separation. The total information provided by the pair increases the farther the pair of cells are spaced apart. This is primarily attributable to an increase in the independent component of the information (means for pairs located $<40 \mu \mathrm{m}$ from each other mediolaterally were significantly different from those with $>40$ $\mu \mathrm{m}$ separation; $p=0.029$; $t$ test). This situation can occur if signal correlation between the cells (i.e., correlation attributable to common tuning) decreases with distance apart. No significant effect of distance on the contribution of either spontaneous synchrony or the stimulus dependence of synchrony to the information was apparent. This suggests that strong distance dependence in the underlying correlation variables can in some cases be very much reduced in terms of impact on sensory coding, an effect not expected a priori.

Spatial correlations can in principle either increase or decrease the information provided by a neuronal population, but the fact that they lead to a decrease under conditions in which the stimulus tuning is similar in the spatial locality (as discussed above) implies that, in this case, the system operates in a redundant coding regimen (Panzeri et al., 1999). This provides additional evidence against a combinatorial spatial pattern code for sensory information, a signature of which would be a positive (synergistic) contribution to population coding by correlations. Figure $5 E$ captured this explicitly by comparing the total pattern information available from an ensemble to the sum of the information values provided by each constituent cell. In all ensembles examined, the pattern information is below the sum, indicating redundant coding between the cells examined. Quantifying this as the fractional redundancy (see Materials and Methods), we examined the relationship between redundancy and the mediolateral distance between Purkinje cell dendrite pairs (Fig. 6C). Redun- dancy was highest between nearby pairs of cells, falling off significantly with distance (correlation coefficient, $r=-0.26 ; p=$ 0.018; $n=80$ pairs). Spike count redundancy was higher than spike pattern redundancy, suggesting again that taking into account the spatial origin of CS events is important for efficient sensory coding.

\section{Discussion}

We have taken advantage of the spatial resolution offered by in vivo two-photon microscopy to reveal that neighboring Purkinje cells in cerebellar cortex can exhibit tightly synchronized CS activity. This spontaneous CS synchrony falls off over $\sim 200 \mu \mathrm{m}$ mediolaterally in cerebellar cortex and is increased by sensory stimulation and harmaline administration. Because harmaline acts to enhance subthreshold oscillations in the inferior olive neurons, which give rise to the climbing fibers (de Montigny and Lamarre, 1973; Llinás et al., 1974), this suggests that local CS synchrony originates in the inferior olive, consistent with studies of climbing fiber connectivity (Sugihara et al., 2001, 2007). Our results, obtained using a technique affording much higher spatial resolution than previously available, are in close agreement with and extend previous studies using multielectrode arrays (Yamamoto et al., 2001; Blenkinsop and Lang, 2006). This local synchrony, in conjunction with subthreshold oscillations at $\sim 10$ $\mathrm{Hz}$ in the somatomotor inferior olive (Urbano et al., 2006), provides a set of discrete time points at which the olivocerebellar feedback circuit is updated.

We found that periodic sensory stimulation entrained Purkinje cell CS activity, as demonstrated by more regular interspike interval structure without a significant change in the average firing rate. Although the overall time-averaged level of synchrony 
A
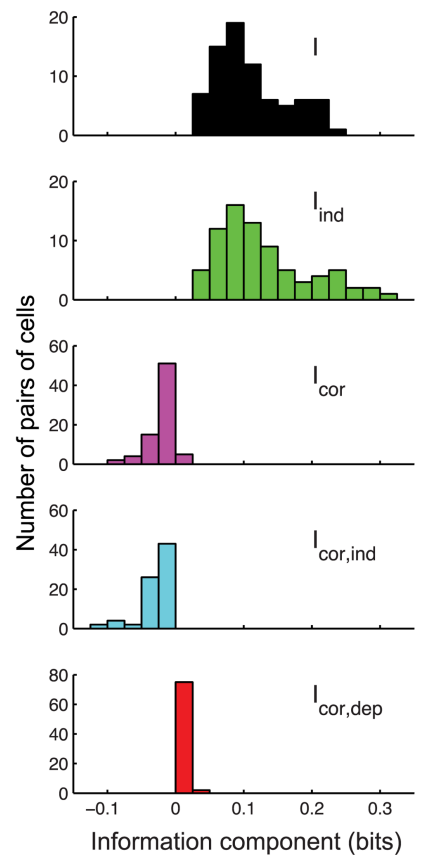

B

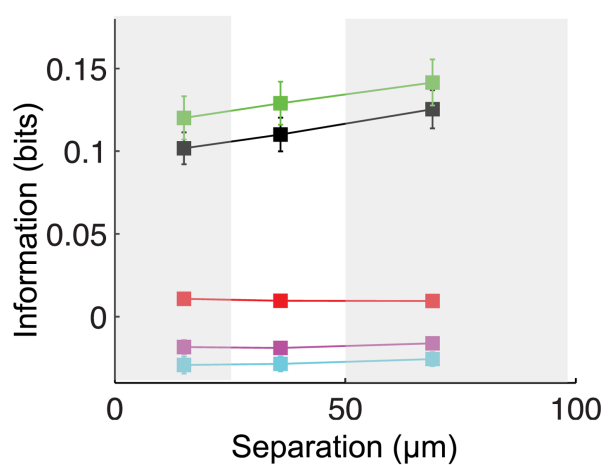

C

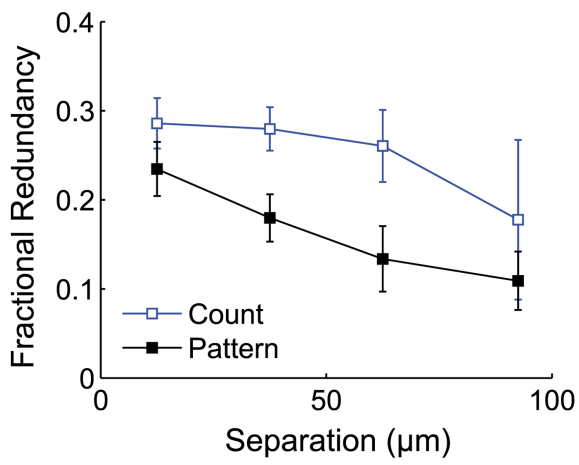

combine the responses of a small pool of Purkinje cells (Palkovits et al., 1977), and it has therefore been proposed that synchrony among DCN inputs could preferentially drive the recipient neurons (Gauck and Jaeger, 2000). Many theories of cerebellar function involve an olivocerebellar feedback loop in which climbing fiber sensory input to the cerebellum is gated by some kind of expectation or error signal (Ito, 2006). Our results suggest that this feedback circuit operates on a population code represented by the spatial pattern of activity in a local set of climbing fibers comprising a microzone. Furthermore, our results indicate that, in that spatial pattern code, sensory signals are represented by a labeled line code: synchronous firing across the pattern does not aid sensory information transmission or discrimination. This leaves open a role for synchrony in flexible selection of certain "lines" for output to the DCN.

Simple spikes in Purkinje cells are invisible to our imaging technique because they generate no detectable dendritic calcium signals (Lev-Ram et al., 1992), but they are of course present in the outputs to DCN neurons. Are DCN neurons required to distinguish between simple and complex spikes to make use of the CS population activity examined here? This is not necessarily the case, because some DCN neurons receive direct inputs from climbing fibers (which convey a signal akin to that we measure here). However, preferential driving by synchronous inputs, as discussed above, could effectively result in was similar under spontaneous and sensory-driven conditions, JPSTH analysis revealed that sensory stimulation modulates the synchrony transiently after stimulus onset. This led us to ask whether DCN neurons receiving small populations of input signals (either complex spikes from Purkinje cells or direct climbing fiber inputs) could make use of the spatial pattern of spikes to indicate whether or when a sensory stimulus occurred. We found that, under our experimental conditions, the pattern of events from ensembles of seven Purkinje cells (the largest ensembles we could systematically examine) provided on average 59\% more stimulus-related information than was available simply by counting the number of complex spikes fired across all cells.

What are the implications of these results for the function of the cerebellar cortical circuit? Our approach effectively measures the sensory population code represented by the climbing fiber outputs of the inferior olive. As well as producing complex spikes (and their associated calcium transients, which we measure) in Purkinje cells, climbing fibers form excitatory synapses with DCN neurons. Purkinje cell connections to DCN neurons are inhibitory; in turn, DCN neurons provide an inhibitory projection to the inferior olive, completing a feedback loop (for review, see Bengtsson and Hesslow, 2006). Thus, there are two routes via which the signals we measure can drive DCN neurons (one of which also carries the simple spikes). Simultaneous recordings from Purkinje cells and DCN neurons suggest that the latter may

the multiplexing of two information channels in the DCN inputs. The complex spike modulates simple spiking of Purkinje cells, e.g., by simple spike pauses after a complex spike (Latham and Paul, 1971; Armstrong and Rawson, 1979), which may in turn elicit rebound firing of DCN neurons (Aizenman and Linden, 1999) (but see Alviña et al., 2008). Synchronous CS activity across Purkinje cells may therefore result in activation of downstream neuronal populations in the DCN, with spatial activation patterns representing information at discrete time points marked out by subthreshold oscillations in the olive.

We have shown that an important aspect of the spatial pattern sensory code is that preserving the identity of the cell producing each CS event (labeled line coding as opposed to pooled coding) is essential to make use of a substantial fraction of the information available. In contrast, second- or higher-order statistical response structure appears to lead to a coding regime in which the information conveyed by neighboring cells within an $\sim 200 \mu \mathrm{m}$ wide region is partially redundant. To make use of this information, DCN neurons might not simply "integrate and fire" but rather make use of spatially compartmentalized dendritic integration rules (Häusser and Mel, 2003; Williams and Stuart, 2003). We hypothesize that the particular spatial patterns observed here may not be random but rather reflect cell combinations that are most useful for movement control [i.e., local scale “motor synergies" (Welsh and Llinás, 1997)]. 
An important, and related, question to ask is not just whether the spatial pattern of activation provides information beyond that available from a pooled code but whether the source of that information is correlation between Purkinje cells. For instance, one hypothesis might be that synchrony among particular ensembles of neurons could be switched on or off to signal a particular stimulus condition. We used the information component analysis approach (Panzeri et al., 1999; Panzeri and Schultz, 2001; Pola et al., 2003) to address this question quantitatively. If switching of synchrony provides or adds to information content, this should be apparent in the information component capturing stimulus dependence of correlation ( $I_{\text {cor,dep }}$ in our notation), which should provide a significant proportion of the correlational component of the information and of the total information. In our experiments, however, $I_{\text {cor,dep }}$ turned out to be negligible, which would appear to discount this hypothesis, at least as far as analysis of the present sensory variable is concerned.

How does the fact that sensory-evoked CS synchrony does not convey any significant information about presence/absence of a sensory stimulus accord with the intrinsic properties of DCN neurons, which as we have discussed may be preferentially driven by CS synchrony? First, we note that this does not preclude CS synchrony from carrying other information (such as about task or motor configuration). Second, in general terms, the presence of synchrony affects (negatively) the information throughput of a downstream receiver, whether or not it is tuned to detect it (Panzeri et al., 1999). The intrinsic properties of DCN neurons thus emphasize the importance of our result for cerebellar function: because DCN neurons are preferentially driven by synchrony and synchrony reduces sensory information throughput in this system, transmission of high-fidelity sensory signals cannot be the functional role of synchrony. Information selection, rather than information transmission, may therefore be a better way to view the function of CS synchrony.

What is the role of the spontaneous CS synchrony that we have observed? One way in which it might play a role in "tagging" spatial assemblies of neurons is that spontaneous synchrony over some time interval might define a functional circuit, within which sensory information is encoded via a labeled line spatial pattern code. In this case, synchrony does not act as part of the coding strategy per se but rather plays an important functional role in establishing/maintaining a dynamic and flexible circuit configuration within which the sensory code is represented. Synchronous ensembles would in this view change according to motor/task configuration rather than according to sensory input received (Welsh et al., 1995; Welsh and Llinás, 1997; Welsh, 2002). By selecting certain labeled lines for output to the DCN, synchrony might effectively act to discard sensory information that is superfluous to the current task. The downside of using synchrony in such a way is a small reduction in the information that can be transmitted per fiber attributable to redundancy; however, redundancy itself may be a feature because it can allow some degree of fault-tolerance or error-correcting behavior $(\mathrm{Pu}-$ challa et al., 2005; Montani et al., 2007).

The neural coding mechanisms examined here are spatially precise, with many defining features falling off significantly over a scale of the order of $200 \mu \mathrm{m}$ mediolaterally, confirming the prediction of cerebellar microzones made three decades ago (Andersson and Oscarsson, 1978). Understanding the fine-scale structure of the sensory code used within a cerebellar microzone thus crucially requires the spatial resolution provided by twophoton microscopy. Our results should lead to many additional lines of investigation: in particular, simultaneous calcium imag- ing from multiple Purkinje cells and electrophysiological recording from DCN neurons would allow DCN synaptic integration and "decoding" properties to be examined directly. In addition, detailed compartmental modeling of DCN neurons, together with electrophysiological investigation of their properties (Gauck and Jaeger, 2003), would allow the hypothesis that the CS spatial population code can be read out by a receiver to be tested. Finally, improved multiphoton imaging acquisition rates may allow the effects observed here to be more specifically related to the $10 \mathrm{~Hz}$ subthreshold rhythm that is a prominent feature of olivocerebellar dynamics.

\section{References}

Aertsen AM, Gerstein GL, Habib MK, Palm G (1989) Dynamics of neuronal firing correlation: modulation of “effective connectivity.” J Neurophysiol 61:900-917.

Aizenman CD, Linden DJ (1999) Regulation of the rebound depolarization and spontaneous firing patterns of deep nuclear neurons in slices of rat cerebellum. J Neurophysiol 82:1697-1709.

Alviña K, Walter JT, Kohn A, Ellis-Davies G, Khodakhah K (2008) Questioning the role of rebound firing in the cerebellum. Nat Neurosci 11:1256-1258.

Andersson G, Oscarsson O (1978) Climbing fiber microzones in cerebellar vermis and their projection to different groups of cells in the lateral vestibular nucleus. Exp Brain Res 32:565-579.

Armstrong DM, Rawson JA (1979) Activity patterns of cerebellar cortical neurones and climbing fibre afferents in the awake cat. J Physiol 289:425-448.

Bell CC, Kawasaki T (1972) Relations among climbing fiber responses of nearby Purkinje cells. J Neurophysiol 35:155-169.

Bengtsson F, Hesslow G (2006) Cerebellar control of the inferior olive. Cerebellum 5:7-14.

Blenkinsop TA, Lang EJ (2006) Block of inferior olive gap junctional coupling decreases Purkinje cell complex spike synchrony and rhythmicity. J Neurosci 26:1739-1748.

Brown IE, Bower JM (2002) The influence of somatosensory cortex on climbing fiber responses in the lateral hemispheres of the rat cerebellum after peripheral tactile stimulation. J Neurosci 22:6819-6829.

Chadderton P, Margrie TW, Häusser M (2004) Integration of quanta in cerebellar granule cells during sensory processing. Nature 428:856-860.

de Montigny C, Lamarre Y (1973) Rhythmic activity induced by harmaline in the olivo-cerebello-bulbar system of the cat. Brain Res 53:81-95.

Gao W, Chen G, Reinert KC, Ebner TJ (2006) Cerebellar cortical molecular layer inhibition is organized in parasagittal zones. J Neurosci 26:8377-8387.

Gauck V, Jaeger D (2000) The control of rate and timing of spikes in the deep cerebellar nuclei by inhibition. J Neurosci 20:3006-3016.

Gauck V, Jaeger D (2003) The contribution of NMDA and AMPA conductances to the control of spiking in neurons of the deep cerebellar nuclei. J Neurosci 23:8109-8118.

Gawne TJ, Kjaer TW, Hertz JA, Richmond BJ (1996) Adjacent visual cortical complex cells share about $20 \%$ of their stimulus-related information. Cereb Cortex 6:482-489.

Häusser M, Mel B (2003) Dendrites: bug or feature? Curr Opin Neurobiol 13:372-383.

Holt GR, Softky WR, Koch C, Douglas RJ (1996) Comparison of discharge variability in vitro and in vivo in cat visual cortex neurons. J Neurophysiol 75:1806-1814.

Ito M (1984) The cerebellum and neural control. New York: Raven.

Ito M (2006) Cerebellar circuitry as a neuronal machine. Prog Neurobiol 78:272-303.

Lamarre Y, de Montigny C, Dumont M, Weiss M (1971) Harmalineinduced rhythmic activity of cerebellar and lower brain stem neurons. Brain Res 32:246-250.

Lang EJ, Sugihara I, Welsh JP, Llinás R (1999) Patterns of spontaneous Purkinje cell complex spike activity in the awake rat. J Neurosci 19:2728-2739.

Latham A, Paul DH (1971) Spontaneous activity of cerebellar Purkinje cells and their responses to impulses in climbing fibres. J Physiol 213:135-156.

Lev-Ram V, Miyakawa H, Lasser-Ross N, Ross WN (1992) Calcium tran- 
sients in cerebellar Purkinje neurons evoked by intracellular stimulation. J Neurophysiol 68:1167-1177.

Llinás R, Sasaki K (1989) The functional organization of the olivo-cerebellar system as examined by multiple Purkinje cell recordings. Eur J Neurosci 1:587-602.

Llinás R, Yarom Y (1986) Oscillatory properties of guinea-pig inferior olivary neurones and their pharmacological modulation: an in vitro study. J Physiol 376:163-182.

Llinás R, Baker R, Sotelo C (1974) Electrotonic coupling between neurons in cat inferior olive. J Neurophysiol 37:560-571.

Lou JS, Bloedel JR (1992) Responses of sagittally aligned Purkinje cells during perturbed locomotion: synchronous activation of climbing fiber inputs. J Neurophysiol 68:570-580.

Marshall SP, Lang EJ (2004) Inferior olive oscillations gate transmission of motor cortical activity to the cerebellum. J Neurosci 24:11356-11367.

Montani F, Kohn A, Smith MA, Schultz SR (2007) The role of correlations in direction and contrast coding in the primary visual cortex. J Neurosci 27:2338-2348.

Montemurro MA, Panzeri S, Maravall M, Alenda A, Bale MR, Brambilla M, Petersen RS (2007) Role of precise spike timing in coding of dynamic vibrissa stimuli in somatosensory thalamus. J Neurophysiol 98:1871-1882.

Nemenman I, Bialek W, de Ruyter van Steveninck R (2004) Entropy and information in neural spike trains: progress on the sampling problem. Phys Rev E Stat Nonlin Soft Matter Phys 69:056111.

Nirenberg S, Carcieri SM, Jacobs AL, Latham PE (2001) Retinal ganglion cells act largely as independent encoders. Nature 411:698-701.

Palkovits M, Mezey E, Hámori J, Szentágothai J (1977) Quantitative histological analysis of the cerebellar nuclei in the cat. I. Numerical data on cells and on synapses. Exp Brain Res 28:189-209.

Panzeri S, Schultz SR (2001) A unified approach to the study of temporal, correlational, and rate coding. Neural Comput 13:1311-1349.

Panzeri S, Schultz SR, Treves A, Rolls ET (1999) Correlations and the encoding of information in the nervous system. Proc Biol Sci 266:1001-1012.

Pola G, Thiele A, Hoffmann KP, Panzeri S (2003) An exact method to quantify the information transmitted by different mechanisms of correlational coding. Network 14:35-60.

Pologruto TA, Sabatini BL, Svoboda K (2003) ScanImage: flexible software for operating laser scanning microscopes. Biomed Eng Online 2:13.

Puchalla JL, Schneidman E, Harris RA, Berry MJ (2005) Redundancy in the population code of the retina. Neuron 46:493-504.

Reidl J, Starke J, Omer DB, Grinvald A, Spors H (2007) Independent component analysis of high-resolution imaging data identifies distinct functional domains. Neuroimage 34:94-108.
Sasaki K, Bower JM, Llinás R (1989) Multiple Purkinje cell recording in rodent cerebellar cortex. Eur J Neurosci 1:572-586.

Schneidman E, Bialek W, Berry MJ 2nd (2003) Synergy, redundancy, and independence in population codes. J Neurosci 23:11539-11553.

Schwarz C, Welsh JP (2001) Dynamic modulation of mossy fiber system throughput by inferior olive synchrony: a multielectrode study of cerebellar cortex activated by motor cortex. J Neurophysiol 86:2489-2504.

Shin SL, Rotter S, Aertsen A, De Schutter E (2007) Stochastic description of complex and simple spike firing in cerebellar Purkinje cells. Eur J Neurosci 25:785-794.

Stosiek C, Garaschuk O, Holthoff K, Konnerth A (2003) In vivo two-photon calcium imaging of neuronal networks. Proc Natl Acad Sci U S A 100:7319-7324.

Sugihara I, Wu HS, Shinoda Y (2001) The entire trajectories of single olivocerebellar axons in the cerebellar cortex and their contribution to cerebellar compartmentalization. J Neurosci 21:7715-7723.

Sugihara I, Marshall SP, Lang EJ (2007) Relationship of complex spike synchrony bands and climbing fiber projection determined by reference to aldolase $\mathrm{C}$ compartments in crus IIa of the rat cerebellar cortex. J Comp Neurol 501:13-29.

Sullivan MR, Nimmerjahn A, Sarkisov DV, Helmchen F, Wang SS (2005) In vivo calcium imaging of circuit activity in cerebellar cortex. J Neurophysiol 94:1636-1644.

Svoboda K, Helmchen F, Denk W, Tank DW (1999) Spread of dendritic excitation in layer $2 / 3$ pyramidal neurons in rat barrel cortex in vivo. Nat Neurosci 2:65-73.

Urbano FJ, Simpson JI, Llinás RR (2006) Somatomotor and oculomotor inferior olivary neurons have distinct electrophysiological phenotypes. Proc Natl Acad Sci U S A 103:16550-16555.

Welsh JP (2002) Functional significance of climbing-fiber synchrony: a population coding and behavioral analysis. Ann N Y Acad Sci 978:188-204.

Welsh JP, Llinás R (1997) Some organizing principles for the control of movement based on olivocerebellar physiology. Prog Brain Res 114:449-461.

Welsh JP, Lang EJ, Suglhara I, Llinás R (1995) Dynamic organization of motor control within the olivocerebellar system. Nature 374:453-457.

Williams SR, Stuart GJ (2003) Role of dendritic synapse location in the control of action potential output. Trends Neurosci 26:147-154.

Wylie DR, De Zeeuw CI, Simpson JI (1995) Temporal relations of the complex spike activity of Purkinje cell pairs in the vestibulocerebellum of rabbits. J Neurosci 15:2875-2887.

Yamamoto T, Fukuda M, Llinás R (2001) Bilaterally synchronous complex spike Purkinje cell activity in the mammalian cerebellum. Eur J Neurosci 13:327-339. 